\title{
Estimación de propiedades mecánicas de materiales metálicos por el método inverso en SPT
}

\author{
Estimation of mechanical properties of \\ metallic materials using inverse \\ method in SPT
}

\author{
Esteban Wenger ${ }^{1}$, Víctor Fierro ${ }^{1}$, Andrea Ansaldi ${ }^{1}$, \\ Rodrigo Gabarain ${ }^{1}$, Nelson Álvarez Villar ${ }^{1}$
}

\author{
${ }^{1}$ Departamento de Mecánica Aplicada - CP: B1603ALO - Juan B. de La Salle 4397, Buenos Aires, Buenos Aires, \\ Argentina \\ e-mail: nalvarezvillar@ citedef.gob.ar
}

\section{RESUMEN}

La caracterización mecánica de materiales metálicos mediante el punzonado de miniprobetas (SPT) permite estimar las propiedades de tracción uniaxial [1]. En este trabajo, por medio de un procedimiento inverso y la simulación numérica de ensayos SPT estimamos las propiedades de tracción uniaxial de acero inoxidable AISI 304L. Detallamos el procedimiento aplicado para estimar la tensión de fluencia, donde se asume la relación de Hollomon de dos parámetros como relación constitutiva. De acuerdo a los resultados obtenidos, se puede sugerir que el procedimiento desarrollado es adecuado para la estimación de las propiedades de tracción en materiales metálicos, adoptando la relación constitutiva adecuada.

Palabras-clave: SPT, FEM, problema inverso, ley potencial de Hollomon.

\begin{abstract}
Mechanical characterization of metallic materials by Small Punch Test (SPT) allows to evaluate uniaxial tensile properties [1]. In this work, by means of an inverse procedure and numerical simulation of SPT tests we estimate uniaxial tensile properties of AISI 304L stainless steel. We detail the applied procedure to estimate the yield stress where the two parameter Hollomon relation is assumed as a constitutive relationship. According to the obtained results, it can be suggested that the developed procedure is suitable for estimating properties in metallic materials, if a suitable constitutive equation is used.
\end{abstract}

Keywords: SPT, FEM, inverse problem, Hollomon's power law.

\section{INTRODUCCIÓN}

Para estimar las propiedades mecánicas, a partir de ensayos de punzonado de miniprobetas (SPT, Small Punch Test) se definen expresiones empíricas para relacionar las cargas sobre la curva experimental con las propiedades mecánicas del material. Con el avance de las técnicas para simulación numérica es conveniente explorar otras posibilidades. Entre ellas, el modelado numérico inverso es una alternativa de interés como HAFEEZ y HUSAIN revisan en [2]. En el método inverso, que se resume en la Tabla 1, se utiliza la curva experimental carga-desplazamiento obtenida en los ensayos SPT y se determinan las propiedades mecánicas del material a partir de la identificación de los parámetros de una ecuación constitutiva tensión-deformación como XU analiza en detalle en [3].

La metodología presentada se aplica a materiales metálicos en general y permite derivar los parámetros de la ecuación constitutiva seleccionada a priori, a partir de ensayos de punzonado de miniprobetas. En este tipo de ensayos, la caracterización de un material requiere evaluar un elevado número de variables que afectan las curvas de carga obtenidas experimentalmente, entre ellas el coeficiente de fricción (probeta-matriz y probeta-indentador), las propiedades macromecánicas del material ensayado (módulo elástico, tensión de fluencia, coeficiente de endurecimiento por deformación, etc.) y las propiedades 
micromecánicas asociadas con el daño mecánico.

Tabla 1: Comparativa entre el método directo e inverso en lo referente a la carga de datos.

\begin{tabular}{l|l|l}
\hline DATOS & MÉTODO DIRECTO & MÉTODO INVERSO \\
\hline Entrada & $\begin{array}{l}\text { Módulo de elasticidad del material } \\
\text { Curva tensión deformación plástica real }\end{array}$ & $\begin{array}{l}\text { Curva experimental carga desplazamiento } \\
\text { del ensayo SPT }\end{array}$ \\
\hline Salida & $\begin{array}{l}\text { Curva simulada carga desplazamiento del } \\
\text { ensayo SPT }\end{array}$ & $\begin{array}{l}\text { Módulo de elasticidad del material } \\
\text { Curva tensión deformación plástica real }\end{array}$ \\
\hline
\end{tabular}

En una primera aproximación al problema, se propone caracterizar macromecánicamente el material utilizando únicamente la primera parte de la curva carga-desplazamiento (elástica hasta transición elastoplástica). En ese caso puede considerarse que los parámetros de daño no tienen influencia por lo que se puede modelar la respuesta del material considerando únicamente deformación elastoplástica. La descripción del endurecimiento por deformación usando ecuaciones constitutivas ha sido extensamente investigada, y se logra mediante la formulación de curvas tensión-deformación reales obtenibles a partir de ensayos de tracción. En KANG et al. [4] se revisan las expresiones más utilizadas. En trabajos aplicados a aceros inoxidables austeníticos se puede consultar entre otros a LEE et al. [5] y para el análisis de deformación plástica extensiva a toda la curva de carga LI et al. [6]. En nuestro trabajo se asume la ecuación constitutiva de Hollomon de dos parámetros y utilizamos, para la validación de la metodología, resultados obtenidos en ensayos de acero AISI 304L, utilizados por FIERRO et al. [7,8] en la implementación del Método Directo.

\section{MATERIALES Y MÉTODOS}

\subsection{Experimental}

El material ensayado es acero inoxidable AISI 304L, que se caracterizó química, metalúrgica y mecánicamente por técnicas convencionales. Las muestras se extrajeron de una chapa laminada en frío con recocido (solubilizado) de $0,7 \mathrm{~mm}$ de espesor.

Tabla 2: Composición química porcentual, método ASTM E41, remanente Fe. A: acero chapa, B: AISI 304L.

\begin{tabular}{c|c|c|c|c|c|c|c}
\hline & $\mathbf{C}$ & $\mathbf{C r}$ & $\mathbf{N i}$ & $\mathbf{S i}$ & $\mathbf{M n}$ & $\mathbf{P}$ & $\mathbf{S}$ \\
\hline $\mathrm{A}$ & 0,03 & 19,9 & 8 & 0,4 & 1,6 & 0,03 & 0,004 \\
\hline $\mathrm{B}$ & 0,03 & $18-20$ & $8-12$ & 1 & $<2$ & $<0,04$ & $<0,03$ \\
\hline
\end{tabular}

Tabla 3: Propiedades de tracción. Dirección de laminación L y transversal T. $\mathrm{S}_{\mathrm{y}}$ : tensión de fluencia; $\mathrm{S}_{\mathrm{uts}}$ : tensión máxima; $\varepsilon_{\mathrm{F}}$ : deformación a la rotura.

\begin{tabular}{c|c|c|c}
\hline MATERIAL & $\mathbf{S}_{\mathbf{y}}[\mathrm{MPa}]$ & $\mathbf{S}_{\mathbf{u t s}}[\mathrm{MPa}]$ & $\boldsymbol{\varepsilon}_{\mathbf{F}}$ \\
\hline Muestra T & 281,2 & 634,4 & 0,570 \\
\hline Muestra L & 296,0 & 673,6 & 0,496 \\
\hline AISI 304L & 295,0 & 660,0 & 0,560 \\
\hline
\end{tabular}

En la Tabla 2 se indica la composición química, mientras que las propiedades de tracción obtenidas experimentalmente conforme a ASTM E8M se informan en la Tabla 3. La metalografía realizada por pulido mecánico y ataque con agua regia metanólica reveló una microestructura de austenita de tamaño de grano 7 según ASTM E112.

En los ensayos SPT se utilizaron probetas de sección 10x10 mm y espesor nominal 0,50 mm. Las probetas para el ensayo se mecanizaron por electroerosión. Las superficies de la probeta se pulieron utilizando papeles abrasivos hasta grit 600. El espesor de cada probeta se controló mediante un micrómetro en cinco posiciones (una en el centro y una en cada centro de los lados).

Los ensayos se realizaron en una máquina SINTECH 2D/L equipada con un dispositivo donde se fijan las probetas y se aplica la carga de punzonado con una esfera de 2,5 $\mathrm{mm}$ de diámetro. Durante los ensayos se registró en forma continua la carga y se fijó la velocidad de desplazamiento entre cabezales en $1 \mathrm{~mm} / \mathrm{min}$. La 
medición de la deformación de la probeta se realiza con un comparador, teniendo en cuenta el análisis de MORENO et al. [9].

En la Figura 1 se presenta una curva carga desplazamiento típica del ensayo de micropunzonado. Pueden distinguirse cuatro zonas en las que el material se comporta de manera diferente.

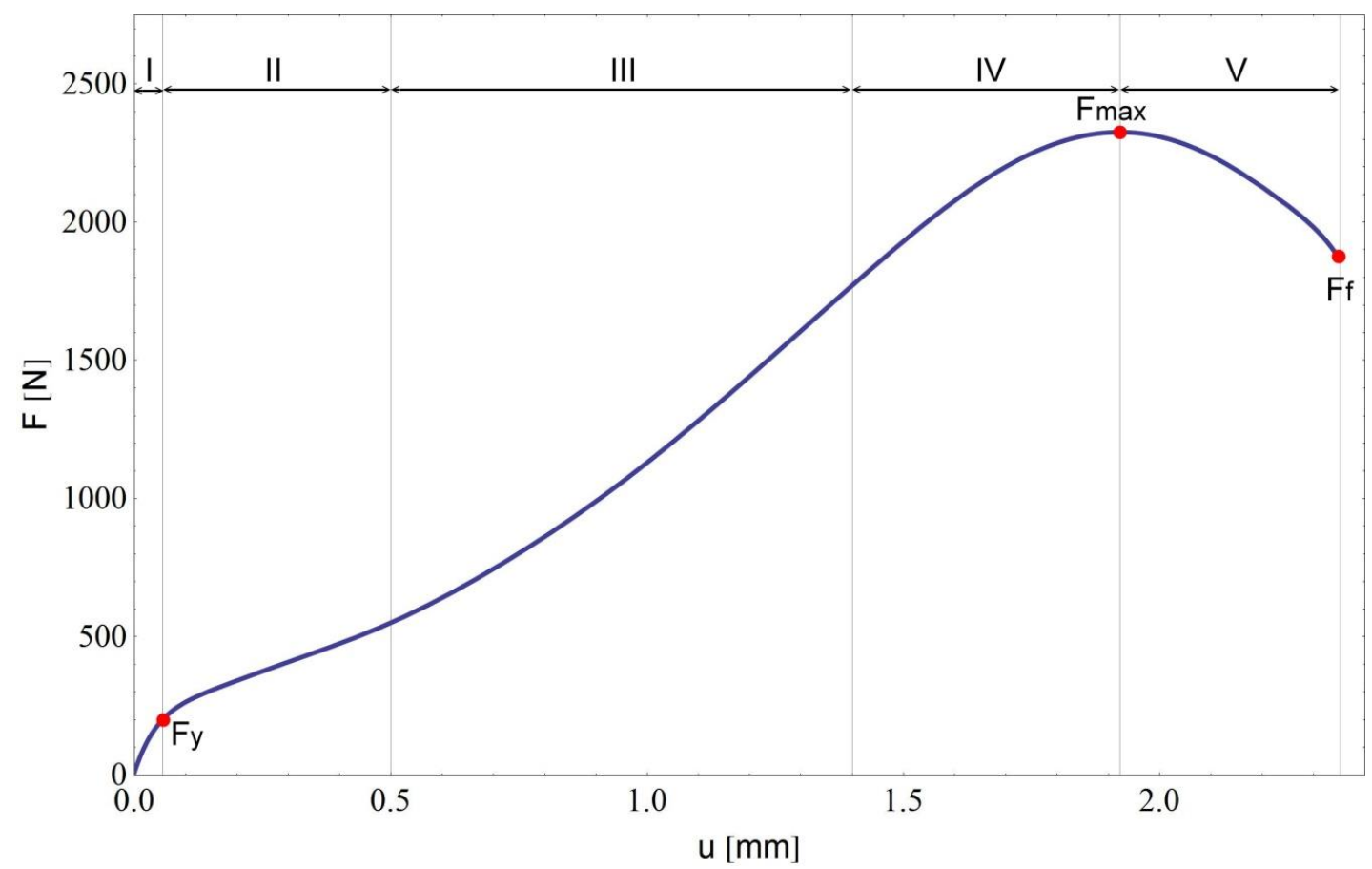

Figura 1: Esquema de curva experimental generalizada. Zona I = Elástica; Zona II = Transición elastoplástica; Zona III = Plasticidad generalizada; Zona IV =Reducción de espesor, Zona V = Carga máxima y posterior fractura.

\subsection{Simulación computacional}

Para la simulación numérica de los ensayos SPT se utilizó el modelo de material multilineal con una ley de endurecimiento tipo Hollomon con parámetros a determinar. En la Figura 2 se detallan las condiciones de contorno del análisis bidimensional axial simétrico realizado. Las partes componentes (eje, indentador, matriz inferior y superior) son sólidos deformables con módulo de elasticidad E $=205 \mathrm{GPa}$ y se mallan por defecto con elementos cuadriláteros mayormente. Debido a que la probeta es sometida a deformaciones relativamente grandes, es necesario realizar un mallado refinado de la misma, se utilizan elementos cuadriláteros con una distancia de $0,075 \mathrm{~mm}$ entre nodo y nodo. En el modelo se asume que el contacto entre la probeta y la matriz inferior es fijo, para simular el apriete perfecto de las matrices. La carga aplicada a la probeta resulta del desplazamiento en el eje vertical del indentador. El contacto entre la probeta y el punzón se ha elegido con un coeficiente de fricción $\eta=0,3$. El modelo fue implementado en ALGOR, FIERRO et al. [1]. 


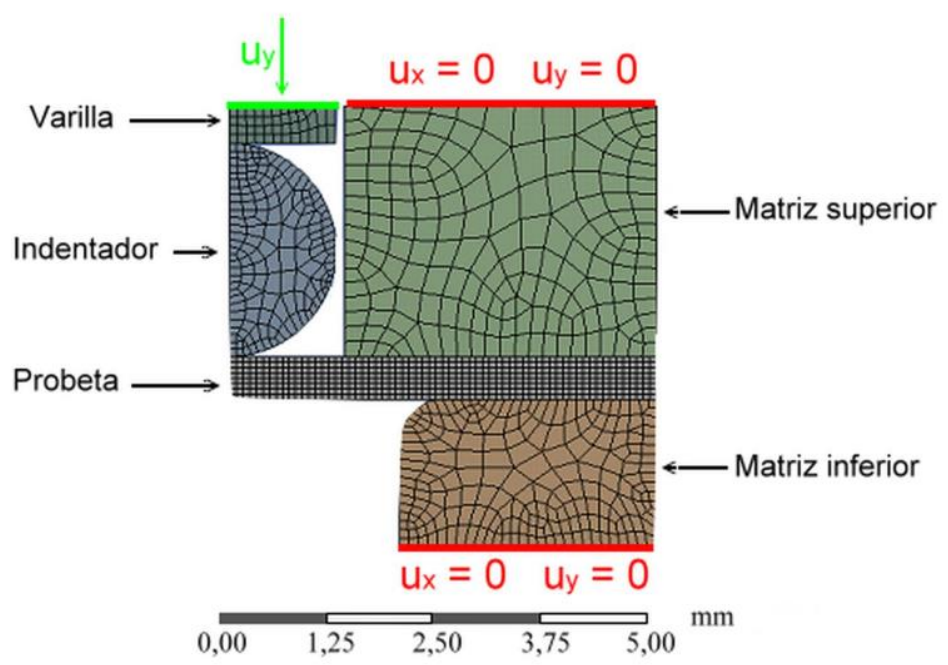

Figura 2: Esquema indicando condiciones de borde y tipo de elementos.

\subsection{Ley constitutiva del material}

En trabajos anteriores se asumió como modelo de plasticidad del material el multilineal con endurecimiento isotrópico, para la simulación de acuerdo al método directo. En ese caso, mediante la simulación se evalúa la sensibilidad de los parámetros del ensayo que no pueden medirse (fricción, carga de sujeción, variación dimensional, etc.) ya que la constitutiva del material es conocida.

En los métodos inversos se trata de asumir constitutivas con parámetros del material que deben ser ajustados. En este trabajo consideramos la ley potencial de Hollomon de dos parámetros $\mathrm{k}_{1}$ y $\mathrm{n}_{\mathrm{s}}$, en concordancia con los trabajos de PEÑUELAS et al. [10,11]. Utilizando esta ley constitutiva es posible obtener los pares de valores reales de tensión - deformación plástica necesarios que definen el modelo. Esta ley ya fue presentada en la ecuación (1), su correspondiente coeficiente de resistencia $\mathrm{k}_{1}$ se presenta en la ecuación (2).

$$
\begin{aligned}
& S=k_{1} \cdot \varepsilon^{n_{s}} \\
& k_{1}=\frac{S_{y}}{\varepsilon_{y}^{n_{s}}} \\
& S \text { tensión total } \\
& \varepsilon_{\text {deformación total }} \\
& S_{y} \text { tensión de fluencia } \\
& \varepsilon_{y} \text { deformación para tensión de fluencia } \\
& n_{s} \text { coeficiente de endurecimiento }
\end{aligned}
$$

Para el ajuste de los parámetros de la ecuación constitutiva de Hollomon, es preciso restringir el análisis donde la deformación plástica domina sobre el daño mecánico. Se requiere que la deformación sea uniforme y el daño mecánico no significativo, que se cumpliría en la primera parte de la curva hasta parte de la zona III, ver Figura 1.

\subsection{Procedimiento de cálculo e iteración}

Los parámetros del material se determinan mediante el proceso iterativo que se indica en la Figura 3.

El ajuste de la curva asume que el material es elástico en la zona I. Conforme a ASTM A 240/A 240M [12], asumimos valores iniciales para el módulo de Young E = $205 \mathrm{GPa}$, coeficiente de Poisson $v=0,30$ y 
coeficiente de endurecimiento $\mathrm{n}_{\mathrm{s} 0}=0,25$.

En la zona II se deben determinar la constante $\mathrm{k}_{1} \mathrm{y}$ el coeficiente de endurecimiento $\mathrm{n}_{\mathrm{s}}$. El cálculo de $\mathrm{k}_{1}$, como lo indica la ecuación (1), está estrictamente vinculado con los valores de $\mathrm{S}_{\mathrm{y}}, \varepsilon_{\mathrm{y}} \mathrm{y}_{\mathrm{s} 0}$. Asumimos un valor medio de $\mathrm{k}_{1}$ de acuerdo al rango de valores presentados por KANG et. al. [4] para los aceros inoxidables. El cálculo de $\mathrm{n}_{\mathrm{s}}$ se realiza a través de la comparación de la curva ensayada contra la simulada de modo análogo al criterio propuesto por YANG et. al. [13].

Se realiza la simulación utilizando como entrada la ley de Hollomon con el parámetro $\mathrm{k}_{1}$ ya determinado y se ajusta el $n_{s}$ partiendo del $n_{s 0}$ inicial e iterando (Figura 3) de modo similar al que utilizan QIAO et. al. [14] hasta que el error calculado de acuerdo a la ecuación (3) sea despreciable.

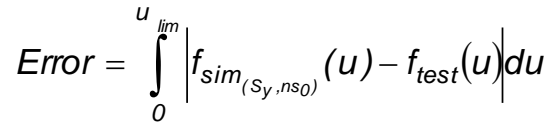

$f_{\text {sim }}(u) \quad$ aproximación polinomial de los resultados de la simulación numérica

$f_{\text {test }}(u) \quad$ aproximación polinomial de las mediciones del ensayo
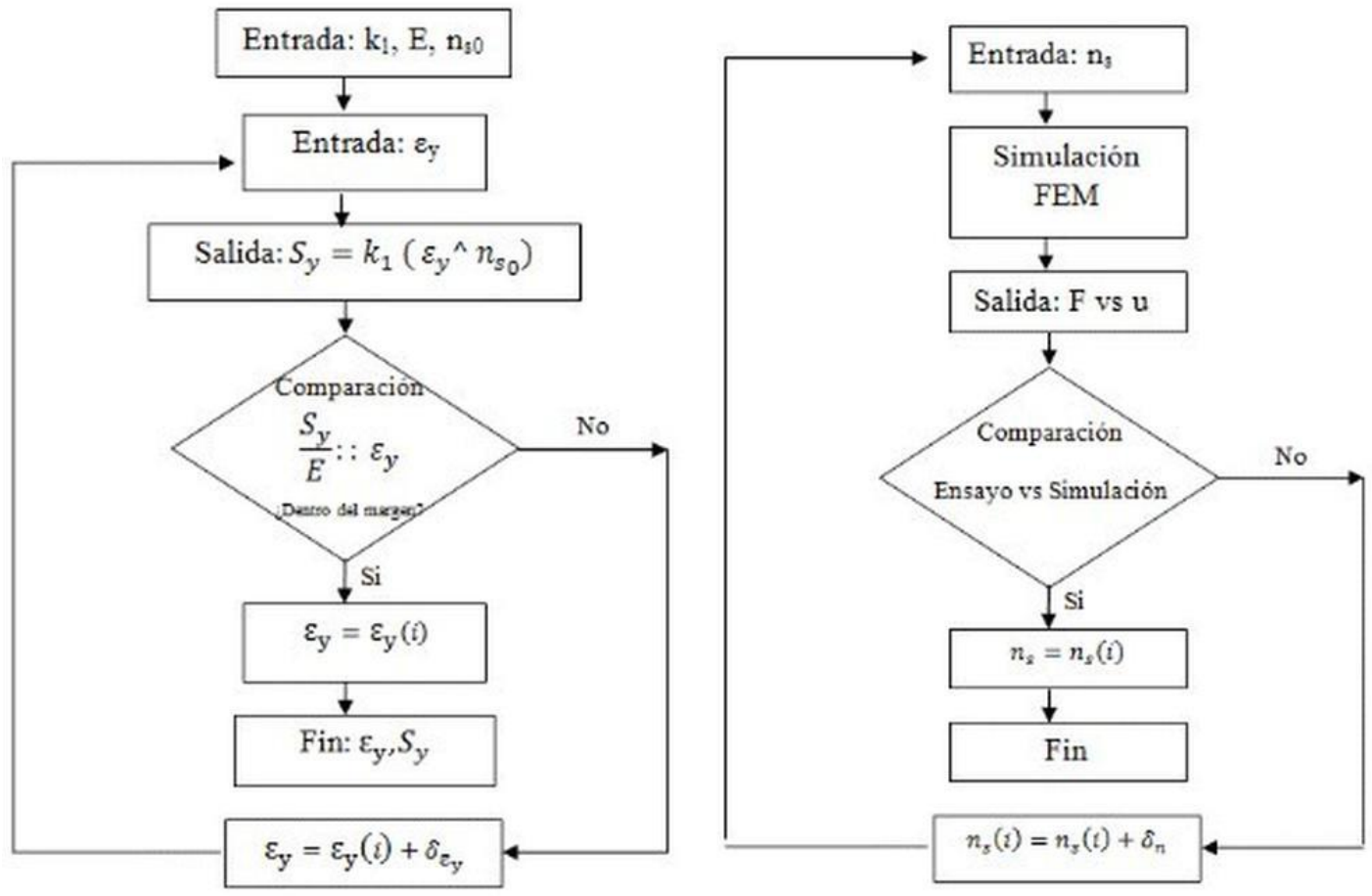

Figura 3: Descripción del proceso iterativo para el cálculo de la constante $\mathrm{k}_{1}$ dentro de la zona $\mathrm{I}$ (parte elástica) y para optimización del coeficiente de endurecimiento $\mathrm{n}_{\mathrm{s}}$ dentro de la zona II y III (parte plástica).

\section{RESULTADOS}

Se han obtenido los parámetros que definen la ley constitutiva de Hollomon, la constante $\mathrm{k}_{1}=1225$ MPa y el coeficiente de endurecimiento $n_{s}=0,29$. En cuanto a las propiedades macromecánicas se ha estimado una tensión de fluencia de $226 \mathrm{MPa}$. La concordancia entre las curvas carga desplazamiento experimental y la simulada puede observarse en la Figura 4. En la Figura 5 se observa la curva simulada con el coeficiente de endurecimiento obtenido por el método inverso y diferentes curvas experimentales.

De la contrastación de los resultados obtenidos con los reportados por KANG et. al. [4] se tiene que el procedimiento aquí utilizado subestima un $6 \%$ el coeficiente de endurecimiento $\mathrm{n}_{\mathrm{s}}$ y la constante $\mathrm{k}_{1}$ de Hollomon, mientras que sobrestima un $12 \%$ la tensión de fluencia (Tabla 4). Por otro lado la diferencia de la tensión de fluencia hallada por el método expuesto, $226 \mathrm{MPa}$, y la obtenida empíricamente por ensayo de tracción convencional, $295 \mathrm{MPa}$, es de un $23 \%$.

Tabla 4: Comparación entre el método publicado por KANG et. al [4] y el propuesto en este trabajo. 


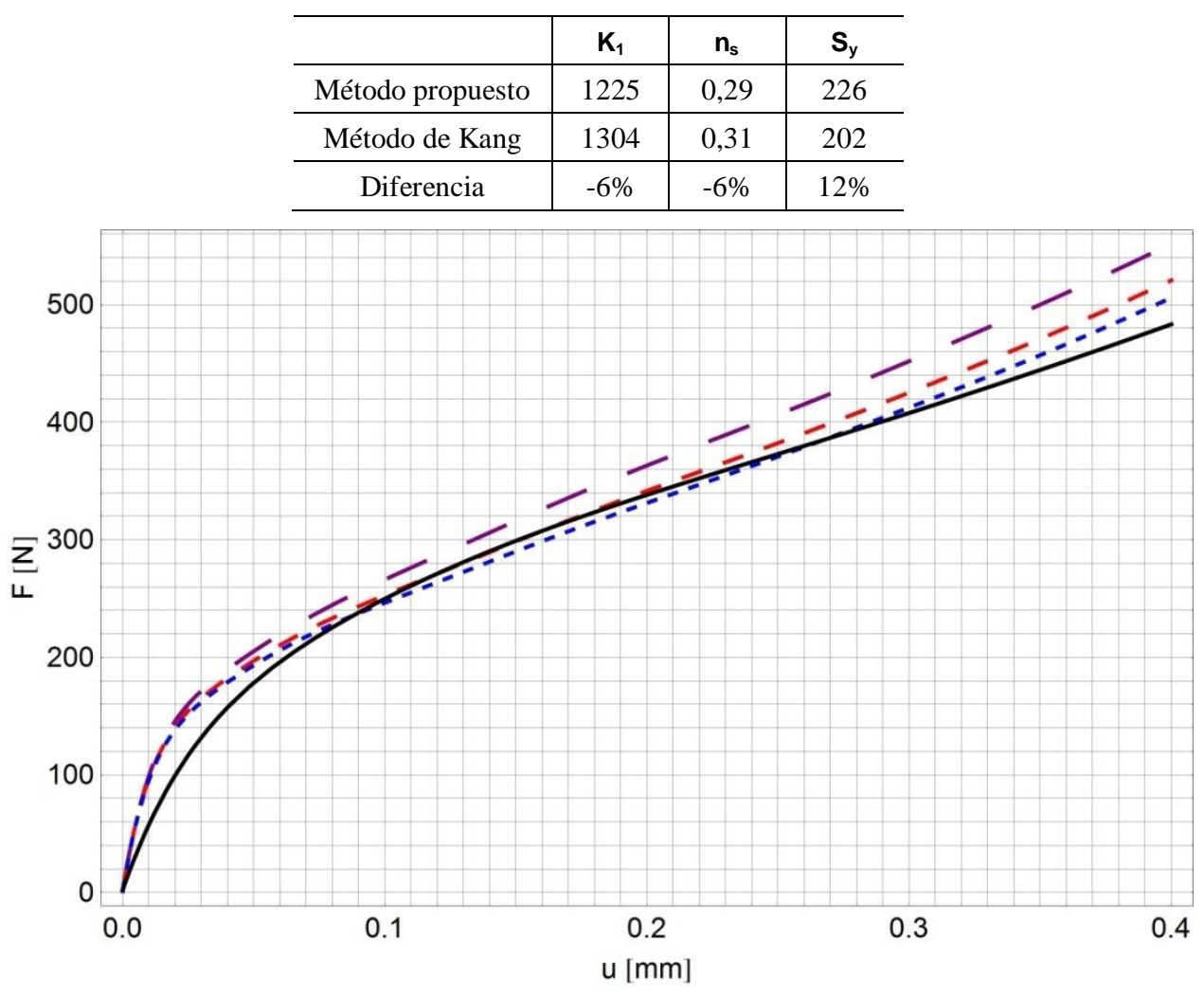

ns $=0.25$ ns $=0.27$ ns $=0.29$ Ensayo

Figura 4: Curvas del ensayo y de la simulación (E=205 GPa; $\left.\mathrm{S}_{\mathrm{y}}=226 \mathrm{MPa} ; \mathrm{k}_{1}=1225 \mathrm{MPa} ; \mathrm{n}_{\mathrm{s}}=0,29\right)$.

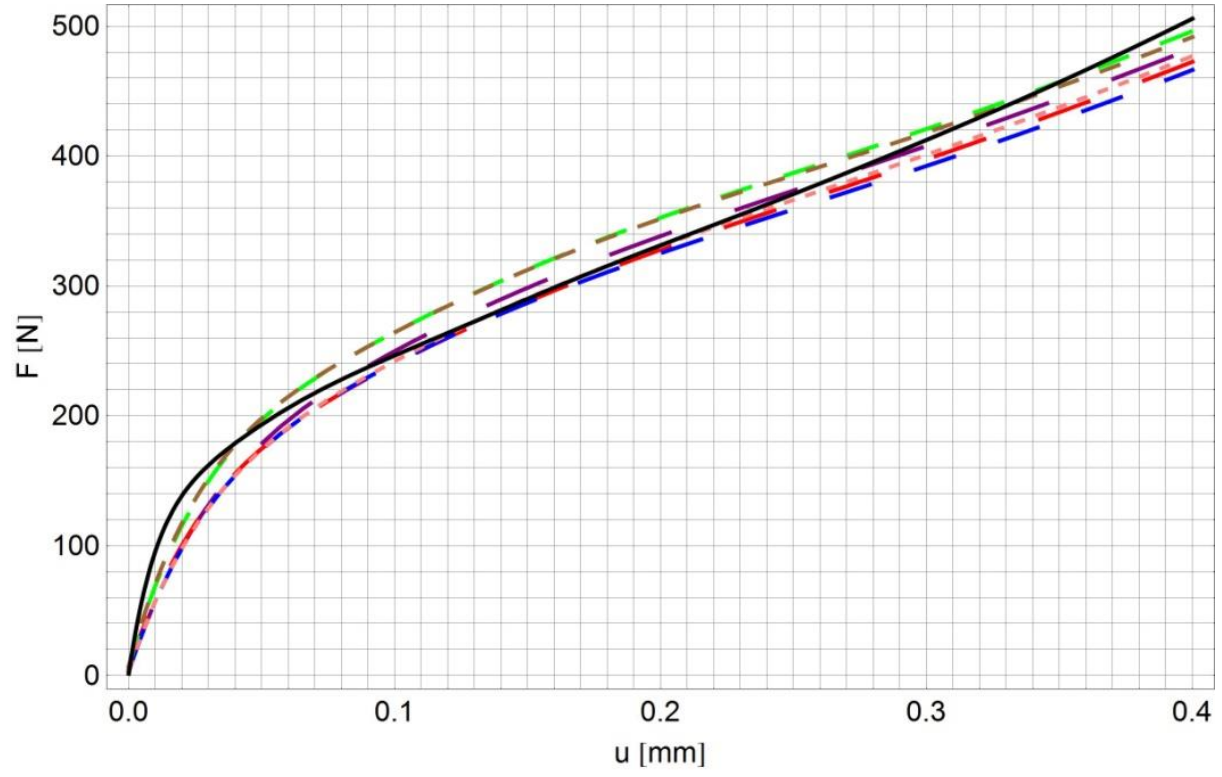

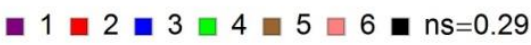

Figura 5: Curvas de carga ensayadas (probetas: 1, 2, 3, 4, 5, 6, de 0,50 mm de espesor) comparadas con la de simulación con coeficiente de endurecimiento $n_{s}=0,29$.

\section{CONCLUSIONES}

Se ha implementado un procedimiento para la obtención de la tensión de fluencia de un material cuyas propiedades se desconocen a partir de una probeta miniatura, ensayándola según el método SPT y resolviendo el problema inverso. Para validarlo utilizamos como material de referencia acero inoxidable AISI 304L. 
La ley potencial de Hollomon de dos parámetros permite predecir con precisión adecuada la tensión de fluencia. La ley constitutiva que describe el comportamiento del material nos permite tener una herramienta analítica contrastable con ensayos experimentales.

La ecuación de Hollomon no describe correctamente el flujo plástico en todo el rango, sino sólo en la zona II (ver Figura 1). En consecuencia no fue posible determinar la tensión máxima.

En esta publicación el objetivo fue estudiar la metodología indirecta de resolución y no analizar las diferentes relaciones constitutivas que describen el endurecimiento del material. En futuros trabajos se prevé aplicar ecuaciones constitutivas alternativas así como considerar daño mecánico, para describir la respuesta del material hasta la fractura.

\section{AGRADECIMENTOS}

Los autores agradecen al Ministerio de Defensa el apoyo económico para el desarrollo del proyecto dentro del programa PIDDEF $\mathrm{N}^{\circ} 16 / 12$.

\section{BIBLIOGRAFIA}

[1] FIERRO, V., ÁlVAREZ VILlAR, N., ANSALDI, A., et al., "Modelado y aplicación del ensayo de punzonado en miniprobetas de acero AISI 304L"; In: Congreso Internacional SAM/CONAMET, Concepción, Chile, 17 al 20 de Noviembre 2015.

[2] HAFEEZ, F., HUSAIN, A., "Diminutive specimen test techniques for predicting mechanical behavior of metals- A review", International Journal for Research in Emerging Sciences and Technology, n. 2, pp.74-90, May 2015.

[3] XU, Z., An inverse finite element analysis and a parametric study of small punch tests, Tesis de Ms. Sc., Universidad de Texas A\&M, College Station, Texas, EE.UU., 2011.

[4] KANG, S.K., KIM, Y.C., KIM, K. H., et al., "Constitutive equations optimized for determining strengths of metallic alloys", Mechanics of Materials, v.73, pp. 51-57, Jan. 2014.

[5] LEE, K.W., KIM, K.H., KIM, J.C., et al., "Derivation of tensile flow characteristics for austenic materials from instrumented indentation technique", Journal of Physics D: Applied Physics, n. 41, pp. 6-11, Mar. 2008.

[6] LI, T., ZHENG, J., CHEN, Z., "Description of full range strain hardening behavior of steels", Springer Plus, 5-1316. In: https://springerplus.springeropen.com/articles/10.1186/s40064-016-3785-x Accedido en septiembre de 2016.

[7] FIERRO, V., ANSALDI, A., GABARAIN, R., Método de obtención de probetas para ensayo SPT (Small Punch Test), Nota Técnica N 932, CITEDEF, Villa Martelli, Buenos Aires, Argentina, 2015.

[8] FIERRO, V., ÁlVAREZ VILlAR, N., ANSALDI, A., et al., Método de obtención y evaluación de propiedades mecánicas en ensayo SPT, Nota Técnica $\mathrm{N}^{\circ}$ 935, CITEDEF, Villa Martelli, Buenos Aires, Argentina, 2016.

[9] MORENO, M.F., BERTOLINO, G., YAWNY, A., "The significance of specimen displacement definition on the mechanical properties derived from Small Punch Test", Materials and Design, v. 95, pp. 623-631, Jan. 2016.

[10] PEÑUElAS, I., BETEGÓN, C., RODRÍGUEZ, C., et al., "Inverse Methods on Small Punch Tests, Numerical Simulations - Applications, Examples and Theory”, Prof. Lutz Angermann (Ed.), 2011, In: http://www.intechopen.com/books/numerical-simulations-applications-examples-therory/inverse-methodson-small-punch-tests Accedido en septiembre de 2016.

[11] PEÑUELAS, I., BETEGÓN, C., RODRÍGUEZ, C., et al., "Determinación de los parámetros elastoplásticos y de daño mediante métodos inversos sobre ensayos SPT", Anales de la Mecánica de la Fractura, v. 24. n. 2, pp. 492-497, Oct. 2008.

[12] ANÔNIMO (2016), "ASTM A240/A240M: Standard Specification for Chromium and ChromiumNickel Stainless Steel Plate, Sheet, and Strip for Pressure Vessels and for General Applications", West Conshohocken, United States, ASTM International.

[13] YANG, S.S., LING, X., QIAN, Y., et al., "Yield strength analysis by small punch test using inverse finite element method", Procedia Engineering, n. 130, pp. 1039-1045, Dec. 2015.

[14] QIAO, J., WANG, W., "Inhomogeneous Material Modeling and Characterization for Aluminium Alloys and Welded Joints, Aluminium Alloys, Theory and Applications", Prof. Tibor Kvackaj (Ed.), 2011, In: 
http://www.intechopen.com/books/aluminium-alloys-theory-andapplications/inhomogeneous-materialmodeling-and-characterization-for-aluminium-alloys-and-welded-joints. Accedido en septiembre de 2016. 